\title{
Mixed-Effect Model of the Fluid Viscosity for Virtual Sensing of the Flow-Front Dynamics
}

Relan, Rishi; Nauheimer, Michael; Madsen, Henrik

Published in:

Proceedings of 2018 IEEE Sensors

Link to article, DOI:

10.1109/ICSENS.2018.8589882

Publication date:

2018

Document Version

Peer reviewed version

Link back to DTU Orbit

Citation (APA):

Relan, R., Nauheimer, M., \& Madsen, H. (2018). Mixed-Effect Model of the Fluid Viscosity for Virtual Sensing of the Flow-Front Dynamics. In Proceedings of 2018 IEEE Sensors (pp. 1-4). IEEE. I E E E Sensors. Proceedings https://doi.org/10.1109/ICSENS.2018.8589882

\section{General rights}

Copyright and moral rights for the publications made accessible in the public portal are retained by the authors and/or other copyright owners and it is a condition of accessing publications that users recognise and abide by the legal requirements associated with these rights.

- Users may download and print one copy of any publication from the public portal for the purpose of private study or research.

- You may not further distribute the material or use it for any profit-making activity or commercial gain

- You may freely distribute the URL identifying the publication in the public portal 


\section{Mixed-Effect Model of the Fluid Viscosity for Virtual Sensing of the Flow-front Dynamics}

Preprint: The original article was presented at the 2018 IEEE Sensors Conference and the original conference

proceedings will be published by IEEEXplore.

\author{
Rishi Relan \\ DTU Compute \\ 2800 Kongens Lyngby, Denmark \\ Email: risre@dtu.dk
}

\author{
Michael Nauheimer \\ Siemens Gamesa Renewable Energy A/S \\ 9220 Aalborg, Denmark \\ Email: michael.nauheimer@siemensgamesa.com
}

\author{
Henrik Madsen \\ DTU Compute \\ 2800 Kongens Lyngby, Denmark \\ Email: hmad@dtu.dk
}

\begin{abstract}
For online control and fault monitoring of the epoxy infusion in a vacuum assisted resin transfer moulding (VARTM) process, good knowledge of the current state of the flow-front is essential. Due to heterogeneous material properties and the environmental conditions, the permeability of the medium, as well as the viscosity of the epoxy, can change significantly during the epoxy infusion process. Hence, for a fast and reasonably accurate estimation of the flow-front, a virtual sensing system capable of combining the physics of the system and the measured data is needed. In this short paper, we propose a data-driven mixedeffect model for the fluid viscosity data acquired from multiple experimental. The proposed model can be easily integrated into the stochastic differential equations (SDE) based virtual sensing system for flow-front dynamics in a VARTM process.

Keywords-Virtual sensing, Mixed-effect model, Viscosity
\end{abstract}

\section{INTRODUCTION}

To reduce the dependency of the fossil fuel requires the inclusion of the renewable energy resources. The European Union has set up a target to increase the use of renewable resources by 2020 to cover around $20 \%$ of its energy needs. Wind energy is one of the primary renewable energy resources in Scandinavian countries as well as in Western Europe, and its capacity is expected to grow by $21 \%$ annually [1]. Therefore, the main emphasis of the wind turbine manufacturing industries is to move towards the Industry 4.0. This transition allows the manufacturing industry to utilize the advancement in sensor technology, data science and digital manufacturing techniques to improve the reliability of the wind turbines as well as the manufacturing process.

Generally, a glass fibre reinforced thermoset polymer is used to manufacture the large-scale composite shell structures like the wind turbine blades using the vacuum assisted resin transfer moulding (VARTM) process. The risk of common moulding defects such as dry spots and voids increases due to inhomogeneous nature of the flow inside the mould. These defect can lead to deterioration of the structure of the blades [2, $3,4]$. Hence, to improve the productivity, structural integrity, quality control and for online control of the manufacturing process, it is essential to monitor the spatiotemporal evolution of the flow-front inside the mould.

Design and development of different sensor technologies for monitoring the VARTM processes is an active field of research. Use of the permittivity sensors [5], pressure sensors [6], electrical time-domain reflectometry sensors [7] and recently developed two-sided visual observation sensors [8] have been reported in the literature. However, at the Siemens Gamesa Renewable Energy factories, the blades are produced in one piece using the patented IntegralBlades ${ }^{\circledR}$ technology [9]. Hence, a visual inspection of the process is not possible. Therefore, the engineers at Siemens Gamesa Renewable Energy are very keen to develop a virtual sensing and monitoring system to improve the control of the manufacturing process.

A grey-box model of the flow-front dynamics based on the stochastic differential equation (SDE) is a good candidate for developing a virtual sensing framework [10]. The virtual sensing framework for the flow-front must consider the change in parameters of the epoxy such as viscosity and permeability during the infusion process. Estimating a good model for the change in viscosity of the epoxy during the infusion process is not trivial $[11,12]$. Therefore to achieve this goal, a stochastic spatiotemporal estimator of the flow-front dynamics with constant viscosity is proposed in $[13,14]$. However, as mentioned before, this may not reflect the reality or observed viscosity change during the manufacturing process. Hence, to emulate the reality, in this paper, we propose to estimate a mixed effect model for the viscosity of an epoxy generally used for laboratory tests at Siemens Gamesa Renewable Energy . The advantage of using the mixed-effect models is that it can accommodate variations in the viscosity measurements due to multiple experiments and varying operating conditions.

The paper is structured as follows: Section II briefly describes the VARTM process. In Section III, SDEs based virtual sensing framework for the flow-front dynamics is discussed. A mixed effect model of the viscosity is proposed in Section IV. Experimental design and the results are discussed in Section 
$\mathrm{V}$, and finally the conclusions are given in Section VI.

\section{THE VARTM PROCESS}

Figure 1 shows a pictorial representation of the work-flow of the VARTM process. The VARTM process is one of the

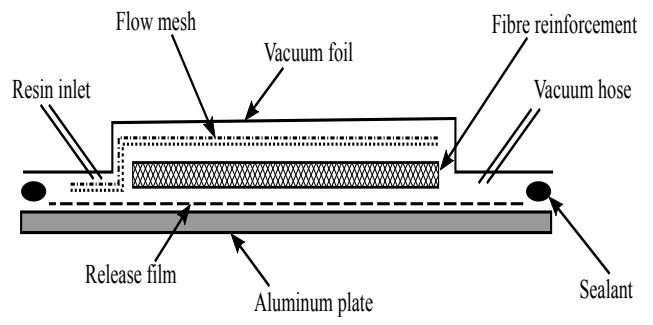

Fig. 1. The work-flow and general material configuration of the VARTM process. As shown above, the epoxy resin enters the vacuum bag through the inlet and further spreads (from left to right direction) into the pores of the fibre reinforcement. This whole process is only assisted by the vacuum generated through the pressure controlled vacuum hose.

special cases of Resin Transfer Moulding (RTM) process. The work-flow during the VARTM process is only controlled by the pressure difference between the near-vacuum inside the mould and the atmospheric pressure of $\approx 1$ bar. The spatiotemporal progression of the flow of the epoxy fluid inside the mould is governed by many factors such as, e.g. the viscosity of the epoxy fluid, the permeability of the material, the porosity of the reinforcement materials, the temperature and the pressure gradient. Notably, the viscosity of the epoxy resin can change during the manufacturing of a long structure like the wind turbine blade due to curing process as well as due to the continuous mixing of new epoxy with the old epoxy in the epoxy reservoir. Furthermore, the viscosity of the epoxy is also temperature dependent. High-temperature results in a low viscosity which implies an increase in cure and flow rates respectively. The epoxy curing process is also an autocatalytic exothermic process resulting in a fast increase of the temperature, especially if the heat cannot escape the blade mould. All these factors make the virtual estimation of the flow-front dynamics a challenging problem.

\section{The Big Picture: SDE based virtual Sensing}

Here, a concise introduction of the spatiotemporal framework utilizing the coupled SDEs approach for virtual sensing of the flow-front inside the mould is given [13, 14]. A coupled SDEs based virtual sensing approach is well suited to estimate the dynamics of stochastic flow-front dynamics inside the mould as it allows us to combine the partial information about the system dynamics with the measured data. The parameters of such virtual sensing model are then estimated directly from the measured data. An SDE based state-space model to describe the flow-front dynamics can be written as [10]:

$$
\begin{aligned}
\mathrm{d} Y_{t} & =f\left(Y_{t}, U_{t}, t, \theta\right) \mathrm{d} t+\sigma\left(U_{t}, t, \theta\right) \mathrm{d} W_{t} \\
Z_{k} & =h\left(Y_{k}, U_{k}, t_{k}, \theta\right)+e_{k}
\end{aligned}
$$

The state equations $Y_{t}$ (which may contain several different states such as the evolution of the flow-front along the line

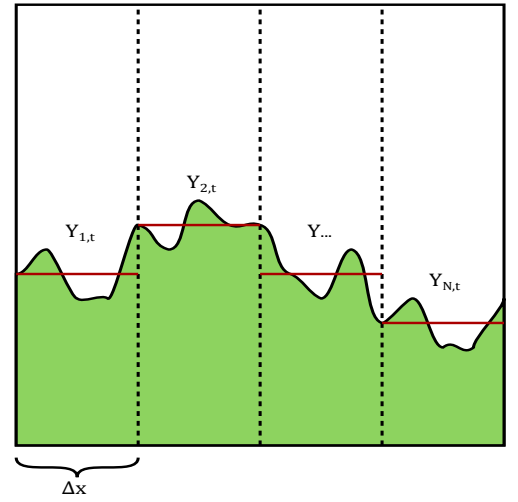

Fig. 2. A pictorial representation of the virtual sensing approach using the coupled SDEs to estimate the flow-front evolution. The flow-front progresses both in space and time along the $y$ direction. The red line shows the domain of spatial dimension $x$ which is approximated using a SDE, whereas the dotted vertical lines show the points of discretisation for the spatial dimension $x$ where the line sensors (such as optical, dielectric sensors etc.) can be placed.

sensors, change in viscosity, and permeability) of the system are formulated in the continuous-time. In SDE formulation the time evolution of the state is separated in a drift term, $f\left(Y_{t}, U_{t}, t, \theta\right)$, and a diffusion term, $\sigma\left(U_{t}, t, \theta\right)$. $W_{t}$ is a Wiener process of dimension $\mathbb{R}^{d}$ with an incremental covariance $Q_{t}$. The discrete-time observations $Z_{k}$ of the observable states are linked to the continuous-time state equation through the observation equation (2). The measurement error $e_{k}$ is assumed to be Gaussian white noise with covariance $\Sigma_{t}$. The inputs are represented by $U_{t}$ whereas $\theta$ represents the parameters of the model. Using the SDE formulation, it is easy to separate the residual error into diffusion (i.e. unmodelled dynamics or the process noise) and the measurement noise, resulting in an accurate description of the prediction error [10].

Figure 2 gives a pictorial representation of the spatiotemporal evolution of the flow-front in a rectangular casting. Here, it is assumed that the flow-front is progressing along a line sensor along the $y$-axis throughout the domain. For estimating the flow-front along the whole $x$-direction, the spatial discretisation is performed using $M_{x}$ grid points. Let $Y_{n, t}$ denote the position of the front at the corresponding value of $x_{m}$ co-ordinate at time $t$. Considering no coupling between neighbouring grid points, the evolution of the flow-front along $N$ lines results in $N$ ordinary differential equations $[13,14]$ :

$$
\frac{d Y_{n, t}}{d t}=\frac{\kappa p_{0}}{\mu} \frac{1}{Y_{n, t}}
$$

Where $p_{0}$ is the pressure, $\kappa$ is the permeability of the porous medium, and $\mu$ represents the viscosity of the epoxy. A coupling factor $D$ is introduced to represent a coupling between the neighbouring lines. Furthermore, to capture the effect of loose coupling (spatial diffusion) between the neighbouring lines as well as any difference between the model and the true system, a constant diffusion term $\sigma$ is introduced for every 
state, which results in [14]:

$\mathrm{d} Y_{n, t}=\left(\frac{\kappa p_{0}}{\mu_{t}} \frac{1}{Y_{n, t}}+D \frac{Y_{n+1, t}-2 Y_{n, t}+Y_{n-1, t}}{\left(\Delta x_{m}\right)^{2}}\right) \mathrm{d} t+\sigma_{n, t} \mathrm{~d} w$

Except for the boundary cases where it is modelled as

$$
\mathrm{d} Y_{1, t}=\left(\frac{\kappa p_{0}}{\mu_{t}} \frac{1}{Y_{n, t}}+D_{b}\left(Y_{2, t}-Y_{1, t}\right)\right) \mathrm{d} t+\sigma_{1, t} \mathrm{~d} w
$$

This formulation has been shown to capture the essential dynamics of flow-front propagation [13, 14]. The major advantage of this SDE based virtual sensing framework is that the change in the viscosity of the epoxy can be easily included by explicitly considering it as an extra state of the model as shown below:

$$
\mathrm{d} \mu_{t}=f_{\mu}\left(t, \theta_{\mu}\right) \mathrm{d} t+\sigma_{\mu_{t}} \mathrm{~d} w
$$

Where the function $f_{\mu}$ can be estimated directly from the data obtained during laboratory tests as shown in the next section.

\section{MIXED-EFFECT MODELLING OF VISCOSITY}

When the observation data from experimental investigation falls into one of $m$ distinct groups $i=1, \cdots, m$, then to accommodate for the groups in a regression model (linear or nonlinear) model, the response $y_{j}$ (here viscosity) in group $i$ is written as:

$$
y_{i j}=\mathcal{G}\left(\phi, x_{i j}\right)+\epsilon_{i j}
$$

Where $j=1, \cdots, n_{i}$ is the number of observations in group $i, x_{i j}$ represents the vector of predictors, $\phi$ is the vector of model parameters, and the measurement error denoted by $\epsilon_{i j}$ is generally assumed to be independent and identically, normally distributed, with constant variance. The parameter vector $\phi$ describe the whole population, i.e. the estimate of $\phi$ is assumed to be the same for all groups. In case the estimates vary with an individual group, the model is described as:

$$
y_{i j}=\mathcal{G}\left(\phi_{i}, x_{i j}\right)+\epsilon_{i j}
$$

In the case of a mixed-effects model, $\phi_{i}=\beta+b_{i}$, where $\beta$ represents the fixed effect and $b_{i}$ represents the multivariate normally distributed random effect, with mean zero and covariance $\psi$. The estimated fixed effects parameter vector $\beta$, as well as the covariance of the random effects $\psi$, describe the whole data set containing different groups where the parameters $\phi_{i}$ are different across groups. Similarly, the estimated random effects parameter vector $b_{i}$ describes specific groups within the data. For the estimation, generally design matrices $\mathcal{A}$ and $\mathcal{B}$ are used for the fixed and random effects parameter identification i.e. $\phi_{i}=\mathcal{A} \beta+\mathcal{B} b_{i}$. Similarly, for accommodating a different design matrices for different groups, the model is reformulated as $\phi_{i}=\mathcal{A}_{i} \beta+\mathcal{B}_{i} b_{i}$. If the design matrices also differ among different observations, then the parameter vector is defined as $\phi_{i} j=\mathcal{A}_{i j} \beta+\mathcal{B}_{i j} b_{i}$ and the model is reformulated as:

$$
y_{i j}=\mathcal{G}\left(\phi_{i j}, x_{i j}\right)+\epsilon_{i j}
$$

Finally, to accommodate the effect of some of the groupspecific predictors $\mathcal{V}_{i}$ in $x_{i j}$, that does not change with observation $j$, the model can be reformulated as:

$$
y_{i j}=\mathcal{G}\left(\phi_{i j}, x_{i j}, \mathcal{V}_{i}\right)+\epsilon_{i j}
$$

In this paper, we used the nlmefit function of MATLAB ${ }^{\circledR}$ to estimate a $3^{r d}$-degree polynomial mixed effect model (function $f_{\mu}$ in (6)) of the viscosity of the epoxy from multiple experiment data categorized by the waiting time.

\section{RESUlTS AND Discussion}

All viscosity measurements were done using a Brookfield CAP 1000 rotational viscometer. Before the mixing of epoxy resin, the viscometer was preheated to a temperature of $30^{\circ} \mathrm{C}$. For the test $100 \mathrm{~g}$ of epoxy resin was transferred into a mixing bucket and the exact weight was noted. After that, a fixed amount of amine hardener was transferred into the mixing bucket, and the exact weight was noted. The exact time (both hour and minute) was noted, and the epoxy was mixed manually for exactly 4 minutes using a countdown timer. After mixing, two-four droplets of mixed epoxy were placed onto the viscometer using a plastic transfer pipette. The viscometer was started, running at a speed of 100 RPM and a shear rate of $200 \mathrm{~s}^{-1}$. The start time was noted and the viscometer was run for $30,60,90,120,150$ and 180 minutes depending on other external test requirements. In total 19 tests were performed, but for this analysis, we randomly selected 6 tests with minimum 120 seconds of waiting time or more.

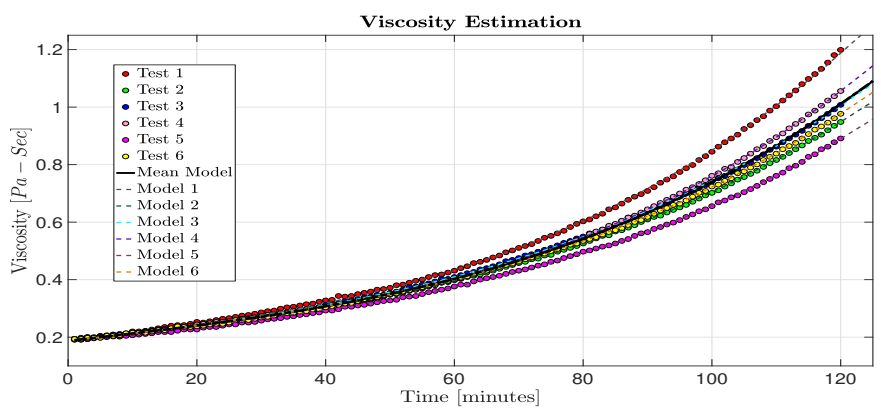

Fig. 3. The figure shows the measurements of the viscosity of the epoxy and the fitted models for different experiments with different waiting times. Dotted circles: Measurement of the viscosity as a function of the waiting time; Black dotted line: Mean viscosity model; Dotted lines: Viscosity models for individual tests

In Figure 3 above, the dotted circles with different colours show the measurement of the viscosity at a certain time point during a particular test. The black dotted line represents the mean viscosity model for all experiments, whereas the dotted lines with different colours represent individual models with different fixed and random effects for different experiments. It can be easily seen that the estimated viscosity model captures both the mean effect due to the increase in waiting time and the random effect due to different tests very well. 


\section{CONCLUSION}

An accurate estimation of the flow-front position is necessary for online monitoring and control of the production process of the wind turbine blades. In this paper, we have proposed a mixed effect model of the viscosity of the epoxy. This mixed effect modelling approach is flexible to also accommodate the effect of temperature as an extra covariate in the model structure. Finally, we also discussed how the estimated model of the viscosity could be easily integrated into an SDE-based virtual sensing framework for the flowfront estimation. In our future research, we will investigate the validity of such an SDE-based virtual sensing framework using the experimental flow-front evolution data.

\section{ACKNOWLEDGMENT}

The authors would like to thank the Manufacturing Academy of Denmark (MADE) for providing the financial support. We thank the laboratory employees at Siemens Gamesa Renewable Energy for their assistance and suggestions.

\section{REFERENCES}

[1] L. Mishnaevsky, P. Brøndsted, R. Nijssen, D. Lekou, and T. Philippidis, "Materials of large wind turbine blades: recent results in testing and modeling," Wind Energy, vol. 15, no. 1, pp. 83-97, 2012.

[2] P. Sreekumar, K. Joseph, G. Unnikrishnan, and S. Thomas, "A comparative study on mechanical properties of sisal-leaf fibre-reinforced polyester composites prepared by resin transfer and compression moulding techniques," Composites Science and Technology, vol. 67, no. 3, pp. 453 - 461, 2007.

[3] C. H. Park, A. Lebel, A. Saouab, J. Brèard, and W. I. Lee, "Modeling and simulation of voids and saturation in liquid composite molding processes," Composites Part A: Applied Science and Manufacturing, vol. 42, no. 6, pp. 658 - 668, 2011.

[4] R. Matuzaki, D. Seto, M. Naito, A. Todoroki, and Y. Mizutani, "Analytical prediction of void formation in geometrically anisotropic woven fabrics during resin transfer molding," Composites Science and Technology, vol. 107, no. Supplement C, pp. 154 - 161, 2015.

[5] B. Yenilmez and E. M. Sozer, "A grid of dielectric sensors to monitor mold filling and resin cure in resin transfer molding," Composites Part A: Applied Science and Manufacturing, vol. 40, no. 4, pp. 476 - 489, 2009.

[6] F. Zhang, B. Cosson, S. Comas-Cardona, and C. Binetruy, "Efficient stochastic simulation approach for $\mathrm{rtm}$ process with random fibrous permeability," Composites Science and Technology, vol. 71, no. 12, pp. 1478 - 1485, 2011.

[7] A. Dominauskas, D. Heider, and J. W. Gillespie, "Electric time-domain reflectometry sensor for online flow sensing in liquid composite molding processing," Composites Part A: Applied Science and Manufacturing, vol. 34, no. 1, pp. $67-74,2003$.
[8] R. Matsuzaki and M. Shiota, "Data assimilation through integration of stochastic resin flow simulation with visual observation during vacuum-assisted resin transfer molding: A numerical study," Composites Part A: Applied Science and Manufacturing, vol. 84, no. Supplement C, pp. 43 - 52, 2016.

[9] H. Stiesdal, P. Enevoldsen, K. Johansen, J. Kristensen, M. Nörtem, and M. Winther-Jensen, "Method for manufacturing windmill blades; Patents: US7473385B2, EP1310351B1," April, 2006.

[10] B. Øksendal, Stochastic Differential Equations: An Introduction with Applications. Springer, 2010.

[11] L. Chang-Lun and W. Kung-Hwa, "Curing kinetics and viscosity change of a two-part epoxy resin during mold filling in resin-transfer molding process," Journal of Applied Polymer Science, vol. 77, no. 10, pp. 2139-2148, 2000.

[12] N. Kiuna, C. Lawrence, Q. Fontana, P. Lee, T. Selerland, and P. Spelt, "A model for resin viscosity during cure in the resin transfer moulding process," Composites Part A: Applied Science and Manufacturing, vol. 33, no. 11, pp. 1497 - 1503, 2002.

[13] M. Nauheimer, R. Relan, U. H. Thygesen, H. Madsen, B. Olesen, K. Kirkeby, and D. Compute, "A stochastic spatio-temporal model of the flow-front dynamics in a vacuum assisted resin transfer moulding process," in accepted in the 18th IFAC Symposium on System Identification, SYSID, vol. 2018, 2018, pp. 1-6.

[14] M. Nauheimer, R. Relan, U. H. Thygesen, and H. Madsen, "Estimation of a stochastic spatio-temporal model of the flow-front dynamics with varying parameters," in accepted in the 9th Conference on "Times of Polymers (TOP) \& Composites”, Ischia - Italy, 2018. 